\title{
A MATEMÁTICA ESTÁ EM TUDO, INCLUSIVE NA GEOGRAFIA
}

\author{
José Aglailton dos Santos Monteiro ${ }^{\mathrm{a}^{*}}$; Jonhatan dos Reis Fernandes ${ }^{\mathrm{a}}$ \\ ${ }^{a}$ Intituto Estadual de Educação, Ciência e Tecnologia do Maranhão. Rua Dom Pedro II, 19, Pindaré Mirim, MA. Brasil. \\ *E-mail para correspondência: geoagla@yahoo.com.br
}

\section{Palavras-chave:}

Geografia;

Cálculos;

Interdisciplinaridade;

Matemática.

\section{Keywords}

Geography;

Calculations;

Interdisciplinarity;

Mathematics.

\section{RESUMO}

O objetivo dessa pesquisa é mostra a relação ou interdisciplinaridade entre a disciplina de Geografia e Matemática no que diz respeito às contribuições matemáticas nos cálculos de fusos de horário, escalas e distancias e coordenadas geográficas, conteúdos muito contestados pelos alunos do primeiro ano do ensino médio, pelo grau de dificuldade. Foi utilizada como metodologia a pesquisa exploratória diante das dificuldades apresentada pelos alunos, questionários e analises de exercícios proposto pelo professor. As análises dos dados demostraram uma fraca base em conhecimentos básicos de matemática, conteúdos que ajudaria a resolver problemas simples como regra de três, divisão e multiplicação nos assuntos geográficos mencionados. Os resultados mostram que devido essa dificuldade inicial, é preciso e, urgente trabalhar a matemática com esses conceitos geográficos afim de sanar essa problemática, uma vez que se faz necessário o conhecimento de tais cálculos para compreensão do espaço e leitura do mundo que se passa pela leitura da matemática, pois está intimamente interligada, intrínseca.

\section{ABSTRACT}

The objective of this research is to show the relationship or interdisciplinarity between the discipline of Geography and Mathematics with respect to mathematical contributions in the calculation of time zones, scales and distances and geographical coordinates, content very contested by students in the first year of high school, by the degree of difficulty. It was used as methodology the exploratory research in face of the difficulties presented by the students, questionnaires and analysis of exercises proposed by the teacher. The analysis of the data showed a weak base in basic knowledge of mathematics, contents that would help to solve simple problems like rule of three, division and multiplication in the mentioned geographical subjects. The results show that due to this initial difficulty, it is necessary and urgent to work on mathematics with these geographical concepts in order to remedy this problem, since it is necessary to know such calculations to understand the space and to read the world that passes by reading of mathematics, because it is intimately interconnected, intrinsic 


\section{INTRODUÇÃO}

A Geografia utiliza a Matemática em seus estudos desde os primórdios da humanidade, onde Erastóstenes de Cirene, considerado o pai da Geografia, usou a matemática para calcular a linha do equador, os trópicos, círculos polares, coordenadas geográficas com seus cálculos, distancia da terra ao sol ou lua, etc.

A Matemática está presente ainda na topografia, cartografia, geomática, fotogrametria, geoestatística, sensoriamento remoto, Processamento Digital de Imagens, análise espacial, Banco de Dados Espaciais, Cadastro Técnico, Sistemas de Informação Geográficos (SIG), mapeamento digital e os sistemas de posicionamento por satélite, como o GPS, Glonass, Galileo.

De acordo com Honda (2013), por meio das possibilidades pedagógicas relacionadas à Geografia e Matemática, pretende-se oferecer reflexões, subsídios, para que o aluno compreenda realmente as situações matemáticas de sala de aula, sugerindo atividades que auxiliem na melhor compreensão, ou seja, que os alunos se envolvam com algo mais atrativo e interessante, sem esquecer-se dos conhecimentos matemáticos a serem compreendidos.

Atualmente, a Matemática dentro da Geografia tornou-se mais relevante para o desenvolvimento dos sistemas da informação geográfica (SIG), essa modelagem matemática de diversos fenômenos na superfície da Terra através do SIG, abriu um importante campo da disciplina, o que permitiu uma maior interação com outros ramos da Geografia como a Hidrologia, Climatologia, Geomorfologia e Geografia Econômica.

Geralmente os conteúdos da Geografia que mais utilizam a matemática são inicialmente tratados, abordando um estudo introdutório da Geografia, para mostrar a localização da Terra no universo e do sistema solar, os movimentos da terra, e a influência do sol e da lua sobre a superfície, destacando cada zona climática do planeta, por exemplo, e à definição e compreensão dos sistemas de localização como a base para todo o estudo geográfico.

É importante comentar a importância da interdisciplinaridade entre as ciências, pois os conhecimentos são interconectados, ajuda-se, se completam. De acordo com Neto (2014), a interdisciplinaridade é um método de pesquisa e de ensino suscetível de fazer com que duas ou mais disciplinas interajam entre si, esta interação podendo ir da simples comunicação das ideias até a integração mútua dos conceitos.

Segundo Luck (2001), o estabelecimento de um trabalho de sentido interdisciplinar provoca, como toda ação a que não se está habituado, sobrecarga de trabalho, certo medo de errar, de perder privilégios e direitos estabelecidos. Diante disso, se faz necessário esse trabalho




interdisciplinar entre as disciplinas, pois, só tem a somar e facilitar o processo ensino aprendizagem, no caso da Geografia e Matemática ambas parceiras de longa data.

É notório que o ensino de matemática pode contribuir e está presente em todas as áreas e, contribui para relações e conexões entre o ensino aprendizagem. Para realizar essa relação, o meio mais seguro é a situação real, usar exemplos práticos de maneira que o aluno veja pra que serve e de que maneira chegar ao resultado de maneira mais fácil.

Sabemos que alguns conceitos básicos da matemática estão presentes na geografia e, mas em todos os professores percebem como poderia ser mais significativo se trabalhassem esses conceitos juntos. Como já foi citado anteriormente: localização de um ponto, ângulo, distâncias entre pontos, conceitos de meridianos e paralelos, de latitude e longitude, arco de circunferência para encontrar as coordenadas geográficas propiciam a relação com escalas, regra de três simples, diâmetro, entre outros, estão presentes na geometria, mas que muitas vezes os professores não chegam nesses conteúdos em matemática até o fim do ano letivo, ou simplesmente pelas dificuldades de ensinar tal conteúdo. Dessa forma, é necessário mais aprofundamento em tal assunto ao invés de se fazer apenas um breve.

Na tabela abaixo, alguns dos conteúdos que tem relação entre Geografia e Matemática.

Quadro 1: Correspondência entre os conteúdos de Matemática e Geografia.

\begin{tabular}{|l|l|}
\hline \multicolumn{1}{|c|}{ MATEMÁTICA } & \multicolumn{1}{c|}{ GEOGRAFIA } \\
\hline ESFERA & GLOBO TERRESTRE \\
\hline COORDENADAS CARTESIANAS & COORDENADAS GEOGRÁFICAS \\
\hline NÚMEROS INTEIROS & FUSOS HORÁRIOS \\
\hline CIRCUNFERÊNCIA MÁXIMA & LINHA DO EQUADOR \\
\hline DISTÂNCIA ENTRE DOIS PONTOS (ARCO) & GEODÉSICAS \\
\hline
\end{tabular}

Fonte: Honda (2013).

De acordo com Libâneo (1994), o processo de ensino se caracteriza pela combinação de atividades do professor e dos alunos, ou seja, de forma que se permita a troca de conhecimentos e os alunos atinjam suas potencialidades e garantam o desenvolvimento de suas habilidades. Assim, a interdisciplinaridade só será eficaz se houver mudança de atitudes em busca da construção de um conhecimento mais global, rompendo com os limites impostos pelas disciplinas, deixando o compartimento do conhecimento. 


\section{METODOLOGIA}

O presente estudo de caráter qualitativo, exploratório e descritivo, foi realizada no Instituto de Educação, Ciência e Tecnologia do Maranhão-IEMA - Unidade Plena de Pindaré Mirim, junto a 30 (trinta) estudantes, numa faixa etária entre 15 (quinze) e 16 (dezesseis) anos. Foram utilizados instrumentos de coleta de dados como análise documental, além de entrevistas e questionários.

Nas etapas para coletas de dados, citam-se: mapeamento dos conteúdos de Geografia, especificamente coordenadas geográficas, cálculos de fuso horário, cálculos de escalas e distâncias, apresentação de métodos de ensino relacionados com ensino de matemática; desenvolvimento da aula em uma turma do ensino médio e avaliação por meio de abordagem oral e atividade escrita em sala de aula. Para análise dos dados, utilizou-se a técnica de análise de conteúdo como técnica qualitativa, e a tabulação para análise dos dados quantitativos.

\section{RESULTADOS E DISCUSSÃO}

Foram aplicados questionários para trinta alunos dos quatro primeiros anos do ensino médio técnico do IEMA - UP Pindaré a fim de sondar as dificuldades prévias dos alunos ou o que mais aflige na Geografia devido a presença a matemática aplicada envolvendo vários cálculos que são precisos na ciência geográfica.

É importante observar pela pesquisa que os alunos têm falta de base da matemática o que impede que se deem bem nos cálculos na geografia e até em outras disciplinas ao qual a matemática está presente.

Foi perguntado para os alunos quantos gostavam das disciplinas de Geografia e de Matemática e, surpreendentemente os resultados foram bem próximos sendo que $90 \%$ gostam de matemática e $80 \%$ de Geografia, ficando apenas $10 \%$ e $20 \%$ de repudio respectivamente. O que chamou atenção e que a maioria diz gostar da disciplina de cálculos sendo que é a mais reclamada por todos, um paradoxo no ar.




Figura 1: Percentual de estudantes que afirmaram gostar da disciplina.



Fonte: Arquivo do autor.

De acordo com os alunos, $75 \%$ aprendem melhor quando os professores apresentam o conteúdo e várias atividades sobre ele e logo em seguida, quando o professor explica várias vezes o mesmo conteúdo.com 16\%, e ainda com $6 \%$ quando o professor apresenta exemplos reais.

Figura 2: Percentual de respostas dos estudantes quanto às metodologias que mais os fazem aprender melhor.

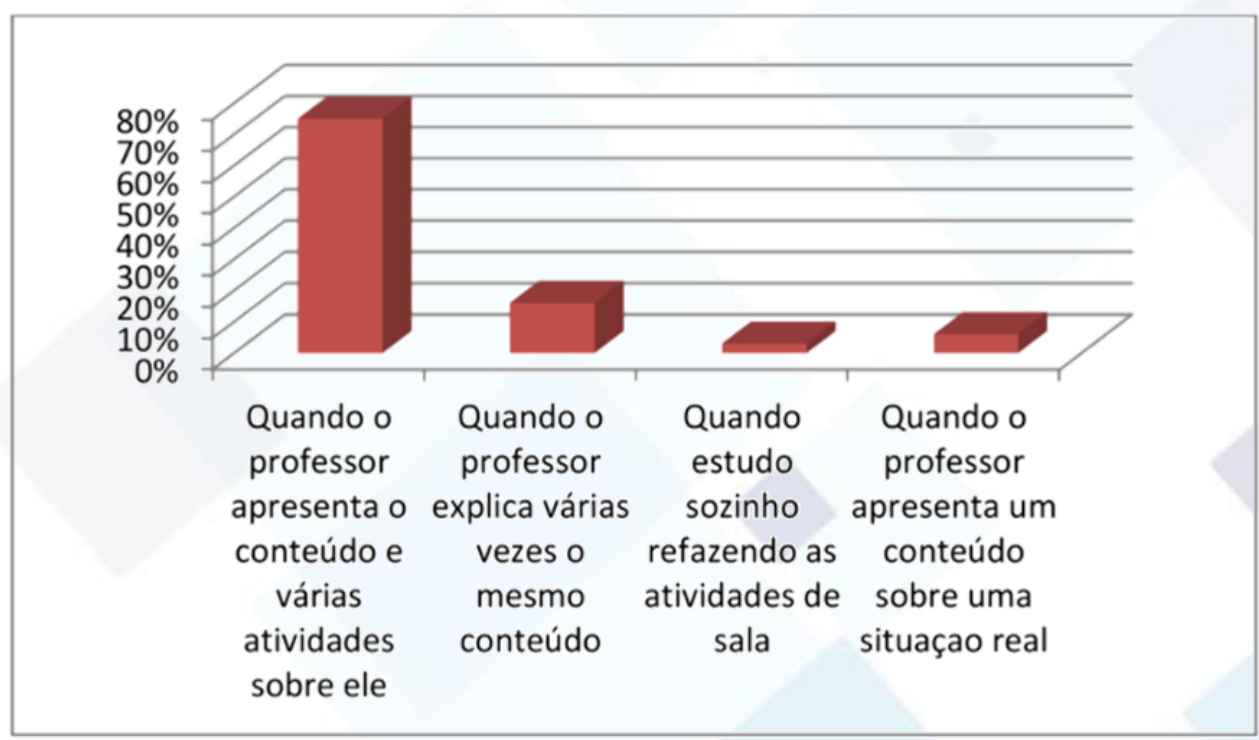

Fonte: Arquivo do autor

Foi perguntando para os alunos o que seria um bom professor na visão de cada um, sendo que a maioria, 96\% disseram que é aquele que sempre inova, que se interessa pelo conhecimento prévio dos alunos, que traz novidades sempre que possível.

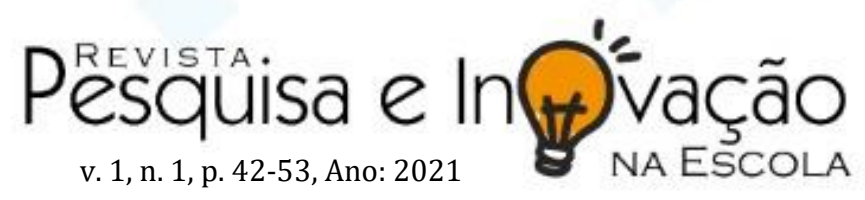


Figura 3: Percentual de repostas dos estudantes quanto ao que eles consideram ser um bom professor.

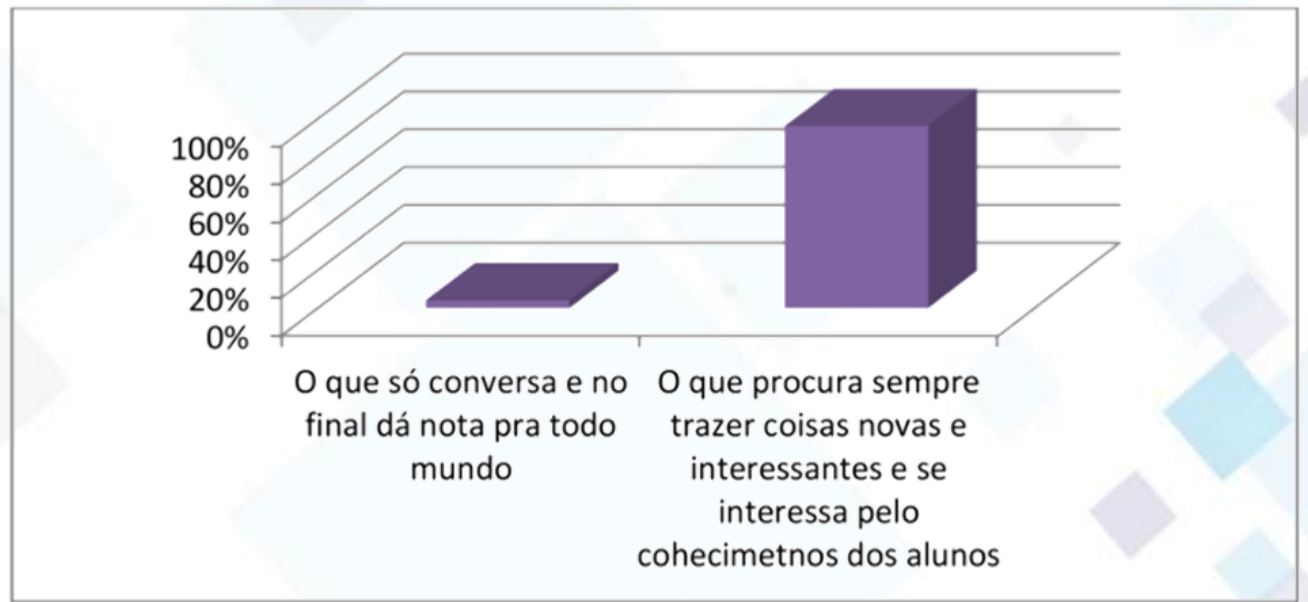

Fonte: Arquivo do autor

A pesquisa também quis saber como são as aulas e, a maioria dos estudantes, em torno de $66 \%$ falaram que as aulas trazem assuntos interessantes para a turma dando oportunidade de participação e colocação do que se sabe sobre o assunto. Uma parcela menor dos docentes, $23 \%$ falaram que preferem as aulas onde se copia e faz atividades para fixação do conteúdo e apenas $11 \%$ consideram as aulas maçantes, sem interesse.

Quando os pesquisados foram perguntados se sabiam o significado da palavra Geografia e Geometria, a maioria não souberam responder com $40 \%$. Já $34 \%$ dos estudantes falaram que geografia é o estudo da terra e geometria o estudo das formas e 13\% responderam que só sabia o significado de geografia e 13\% só sabia que estudava o espaço. Fica evidente, nesse caso, que a maioria dos alunos não sabem ao certo o significado e o que estuda cada um dos temas apresentados, o que nos leva a pensar que falta um pouco mais de atenção por parte dos alunos e também do professor em reforçar com exemplos práticos, sem falar que a base da matemática sempre está presente nessas dificuldades. 
Figura 4: Percentual de estudantes q ue sabem o significado de Geografia e Geometria.

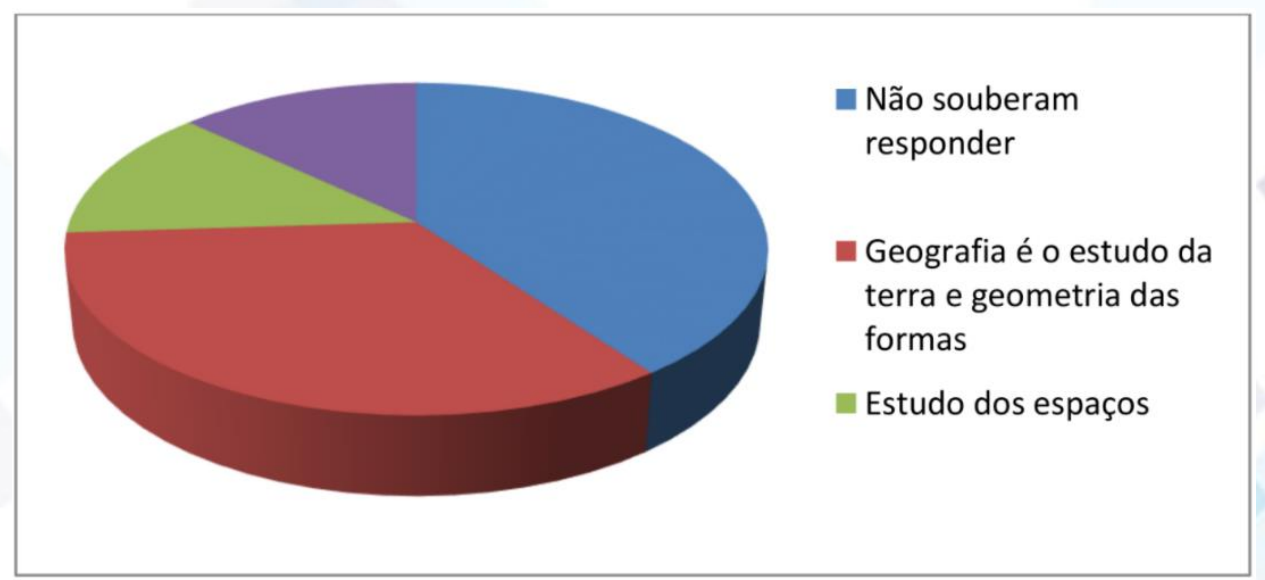

Fonte: Arquivo do autor.

Foi perguntado aos alunos, se conheciam o que era uma esfera e seus elementos, sendo que apenas $30 \%$ responderam saber, enquanto $56 \%$ afirmaram não saber e $7 \%$ responderam mais ou menos e 7\% não responderam. Dessa maneira, observa-se que a base da matemática na geometria também não se faz presente para os estudantes.

Um dos temas de grande valia na geografia é sobe as coordenadas geográficas que servem para o estudo da localização precisa, e quando perguntados sobre isso, $26 \%$ falaram que sabem que são linhas imaginárias, 26\% disseram ajudar na localização, 26\% são as latitude e longitudes, $20 \%$ nada sabem e $22 \%$ mais ou menos. Nesse quesito, percebe-se que a maioria compreende de maneira fragmentado o conceito de coordenadas geográfico.

Figura 5: Respostas dos estudantes sobre o que são coordenadas geográficas.

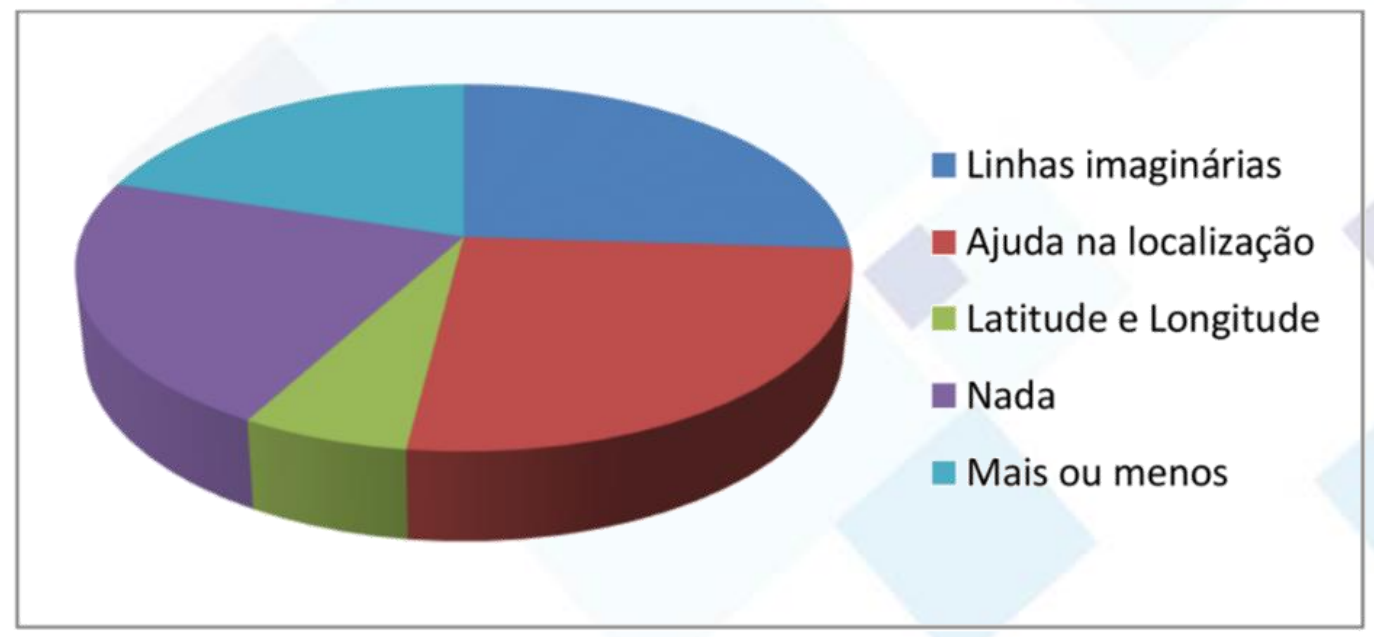

Fonte: Arquivo do autor




Quando questionados sobre a relação entre circunferência, latitude e longitude a maioria afirmaram não saber $64 \%$, outras não souberam responder $10 \%$, e apenas $26 \%$ responderam que seria a distância, ponto e divisão.

Fica evidente a falta de noção sobre a relação intrínseca entre esses elementos que se comunicam e se entrelaçam, que é tão importante para o entendimento de ambos.

Figura 6: Respostas dos estudantes sobre a relação arco de circunferência, latitude e longitude.

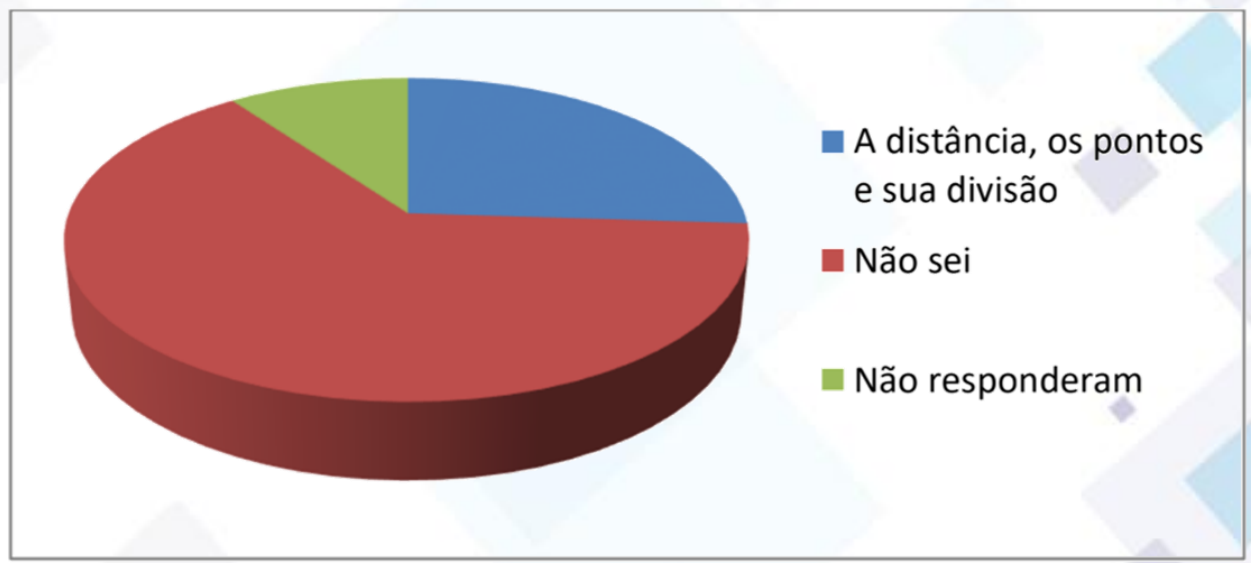

Fonte: Arquivo do autor.

No quesito sobre a resolução de fuso horário, $64 \%$ dos estudantes responderam não saber resolver esses problemas, enquanto apenas 36\% disseram que conseguiriam resolver qualquer problema de fuso. Dessa forma, fica evidente que a base da matemática continua sendo o gerador problema.

Tabela 1: Dificuldade no cálculo de Fuso Horário.

\begin{tabular}{lc} 
Interpretação & $\mathbf{1 0 \%}$ \\
Saber os fusos & $20 \%$ \\
Regras & $10 \%$ \\
Divisão & $30 \%$ \\
Tudo & $14 \%$ \\
Nada & $16 \%$ \\
\hline
\end{tabular}

Fonte: Arquivo do autor 
A tabela acima sobre qual a maior dificuldade em cálculo de fusos confirma o gráfico anterior, pois há dificuldade em não saber interpretar os dados, em não conhecer os fusos, em não saber a regras de fuso horário, e a maioria 30\%, em não saber divisão, umas das operações básicas bases da matemática, o que fica escancarado o problema da maioria dos estudantes do ensino médio, que é a falta de base na matemática.

Foi perguntado para os estudantes se conseguiam resolver problemas envolvendo cálculos de escalas e distâncias, onde mais da metade falaram que sim, enquanto 14\% disseram que não e $33 \%$ disseram saber mais ou menos. Seguindo ainda, quis saber qual a dificuldade nesse tipo de cálculo e a tabela abaixo dá um panorama do problema.

Tabela 2: Dificuldade no cálculo de Escalas e Distâncias Geográficas.

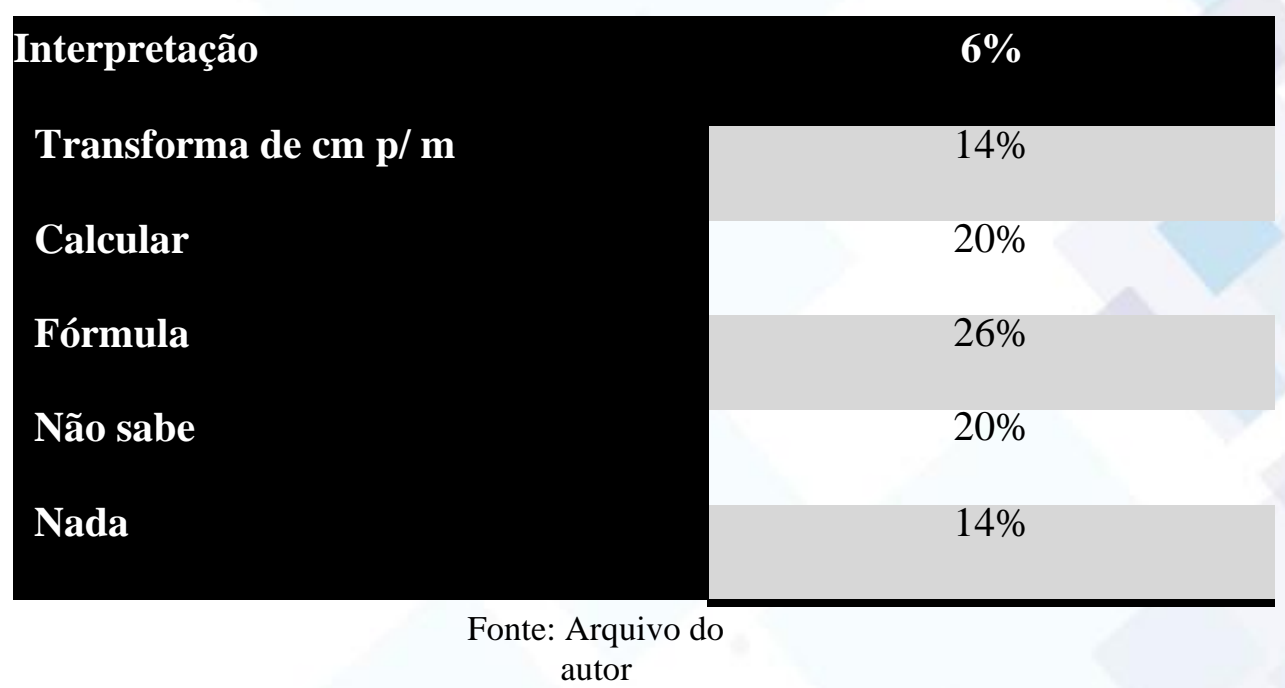

No caso de escalas e distâncias o menor problema é a interpretação, sendo que aprender as fórmulas, calcular e transformar centímetro para metro são as maiores dificuldades, o que fica claro que operações básicas, como multiplicação e divisão que são necessárias para resolver tais cálculos, não foram apreendidos de maneira satisfatória pelos estudantes pesquisados.

Em outra pergunta os estudantes foram questionados se conseguem relacionar o tema projeções cartográficas com conteúdos matemáticos, e a maioria, 54\% confirmaram que sim e, os outros $36 \%$ que não, $10 \%$ relacionam com plano cartesiano. 
Figura 7: Respostas dos estudantes quando perguntados os conteúdos mais difíeis eml Geografia e Matemática.

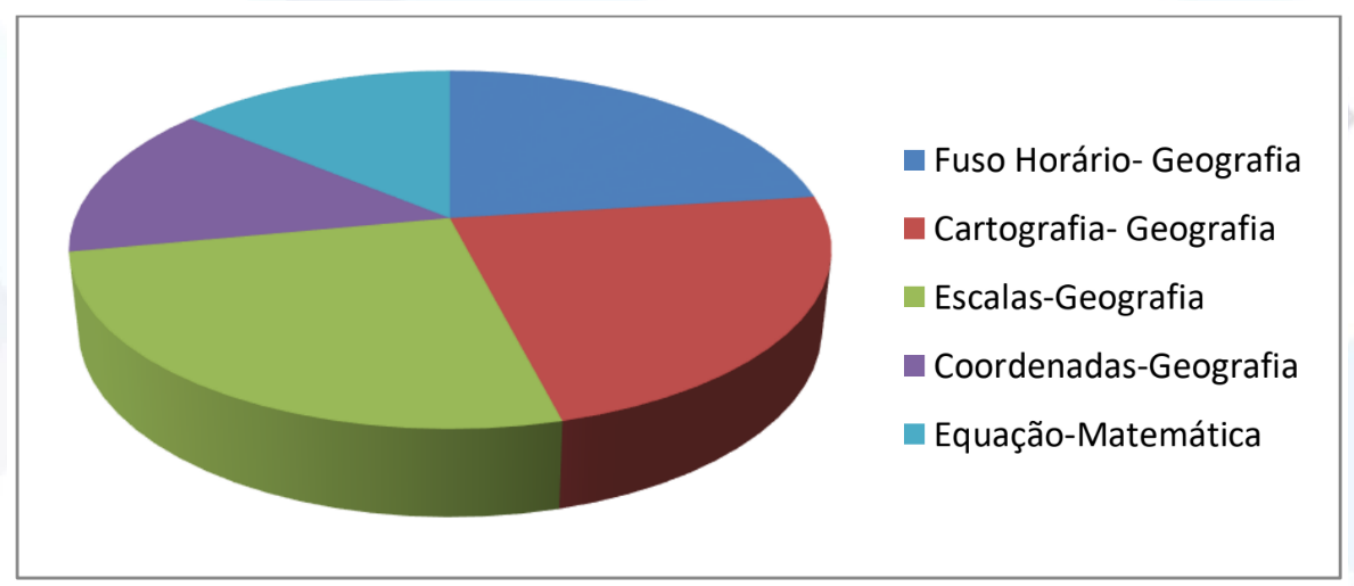

Fonte: Arquivo do autor

Na pesquisa realizada até o final do primeiro semestre, foi perguntado qual o conteúdo mais difícil estudado até aquele momento tanto em geografia, como em matemática e os relacionados foram fuso horário com $23 \%$, escalas $26 \%$, cartografia com $23 \%$, coordenadas geográficas com $14 \%$ e, equações com $14 \%$.

Observa-se que todos os assuntos mencionados precisam da matemática em algum momento, seja para a realização de cálculo, ou outros fatos onde a disciplina dos números se envolvam.

\section{CONSIDERAÇÕES FINAIS}

Diante dos estudos levantados e resultados apresentados fica evidente que a base da matemática, é de suma importância para os conteúdos de geografia aos quais abordam cálculos, como escalas, distâncias, coordenadas geográficas, cálculos demográficos, etc. associado com os conteúdos de matemática como plano cartesiano, arco de circunferência, números inteiros etc.

Daí a importância de reforçar a base matemática com entendimento por exemplo, do múltiplos e submúltiplos do metro e as quatro operações básica da matemática, pois muitas vezes alunos que fazem um fundamental fraco, chegam com essas dificuldades. 


\section{REFERÊNCIAS}

CAVALCANTE, R. N. B.; SOUSA, M. H. R.; DE SOUSA, J. P. R. Localização e distâncias no mapa da cidade: uma conexão entre Matemática e Geografia. Ensino de Matemática, p. $181,2019$.

CAVAlCANTE, R. N. B.; SOUSA, M. H. R.; DE SOUSA, J. P. R. A interdisciplinaridade entre Matemática e Geografia: inferindo conceitos de localização e distâncias na cidade. Revista Encantar-Educação, Cultura e Sociedade, v. 1, n. 3, p. 07-20, 2019.

DOS SANTOS, C. A.; HALBERSTADT, F. F. Uma prática interdisciplinar entre Matemática e Geografia no Ensino Médio Técnico Integrado: construção de países imaginários. Research, Society and Development, v. 9, n. 6, p. 81, 2020.

DE JESUS BRITO, A. A história da matemática na obra Geografia Geral de Bernhard Varenio. Zetetike, v. 14, n. 2, p. 89-102, 2006.

FERRAZ, C. B. O ensino de Geografia para além da geometrização do espaço: apontamentos entre o redondo e as retas. Caderno Prudentino de Geografia, v. 1, n. 23, p. 38-50, 2001.

FERREIRA, C. G. N. O saber da matemática e suas contribuições para o ensino da geografia: A interdisciplinaridade como método de aproximação pedagógica. VI ENID - III ENFOPROF. João Pessoa: UEPB, 2014.

HONDA, A. M. C. Matemática e geografia: uma interdisciplinaridade. Universidade Estadual de Londrina, Centro de Ciências Exatas, Programa de Pós-Graduação em Matemática Londrina, 2013.

LIAO, T.; DE CARVALHO, J. M. J. Realidade Aumentada e Interdisciplinaridade: o Uso do Aplicativo LandscapAR no Ensino de Matemática e Geografia. EaD em Foco, v. 10, n. 2, 2020.

LIBÂNEO, J. C. Didática. São Paulo: Cortez, 1994

LUCK, H. Pedagogia interdisciplinar: fundamentos teórico-metodológicos. 18. ed. Petrópolis: Vozes, 2013.

PONTUSCHKA, N. N.; PAGANELli, T. I.; CACETE, N. H. Para ensinar e aprender geografia. 3.ed. São Paulo: Cortez, 2009.

PRESTES, I. da C. R. et al. Geometria Esférica: Uma conexão com a Geografia. 2006.

ROSA, R. U.; SANTOS JR, D. N.; LAHM, R. A. O recurso das imagens de satélite para estudo do lugar do educando: uma experiência na área da Matemática e da Geografia. Experiências em Ensino de Ciências, v. 2, n. 2, p. 23-36, 2007.

SANTOS, R. F.; DE OLIVEIRA, E. F. Relações entre a matemática, a geografia e a educação física: proposta de uma educação inclusiva e integradora. Horizontes-Revista de Educação, v. 9 , n. 16, p. 1-24, 2020.

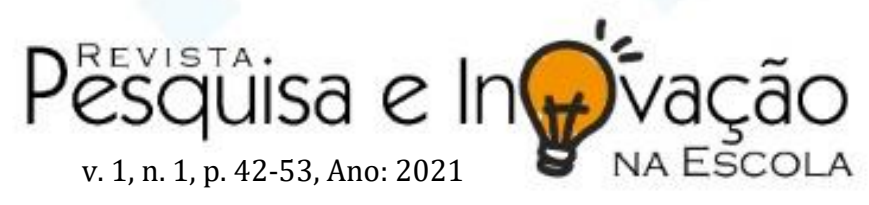


SILVA, J. M.; PEREIRA, M. C.; BERNARDES, S. M. O saber matemático e suas contribuições para o ensino da Geografia. Revista Interdisciplinar Sulear, 2018. 